\title{
Conception of Nan Yin Digital System
}

\author{
F.F. Su \\ College of Computer Science and Technology \\ Huaqiao University \\ Xiamen, China
}

\section{J. Ding}

Nanwei Software Limited Company Quanzhou, China

Abstract--This paper proposes a conception of Nan Yin digital system to protect and inherit Nan Yin well and change the present situation of Nan Yin, which depends on people carriers by means of oral instruction and rote memory. The system includes four points inside: Nan Yin opern digital system, Nan Yin audio digital system, Nan Yin opern auto-play system, constructing the map of Nan Yin digital cultural knowledge and the spatial semantic model by using ontology, so as to complete digital performance on the computer and network. It is convenient for people to search, visit and share, based on improving the value of knowledge.

\section{Keywords- Nan Yin; digital; opern; audio; standard database}

\section{INTRODUCTION}

Nan Yin has been enlisted in Masterpieces of the Oral and Intangible Heritage of Humanity, which is called "living fossil in the history of Chinese music". But today, it faces severe tests of being accepted and continued with difficulty [1].

Near a hundred years Nan Yin had a lot of important achieves, concerning theoretical research, musical literature and academic proof, many of which are creative and significant. However in the country it out of focus in how Nan Yin can be saved and protected completely and organized scientifically, fully and systematically[2].

Digital audio technology has matured [3] and forms an international standard, besides there are experiences of domestic and international mainstream music digital[4]. Domestic research results of Nan Yin provide starting and building up the Chinese Nan Yin digital system project with high quality resources. It can be transformed from oral teaching to the form of digital storage, inheritance and communication, which changed traditional forms and inherited China's traditional culture[5].

\section{CONCEPTION OF NAN YIN DIGITAL SYSTEM}

In order to realize the organization storage of cultural knowledge, the first problem which have to be faced is modeling cultural knowledge representation. Modeling cultural knowledge of cultural space relate to how to express efficiently and how to store efficiently. The Nan Yin digital system completes digital expressing and encoding special Nan Yin music and opern, learning mature digital audio technology and opern technology. So opern(handwritten manuscripts) need to be recognized with image recognition technique and voice need to be recognized with speech recognition technique, then transcribes them into digitized code for long-term storage, transmission and inheritance. Finally we can decode digital Nan Yin music and finish a standard speech synthesis so that Nan Yin music could be played. Besides the system could create opern automatically and Nan Yin opern and voice automatically convert digital terms so that people can search, visit, share and reuse easily, advancing the value of knowledge.

The main contents of Nan Yin digital system include:

\section{A. Nan Yin Opern Digital System}

1) Features, present situation and problems of Nan Yin opern: Nan Yin including "Finger, spectrum, tune" three types. Gongche notation is a special notation used by Nan Yin. Gongche notation forms its own system. As the remaining form of recording ancient music, a word in the spectrum corresponds to that special finger position of instrument. Traditional opern recorded only skeleton. Singsong create real

\author{
C. Wang \\ College of Computer Science and Technology \\ Huaqiao University \\ Xiamen, China
}

J. Gou

College of Computer Science and Technology Huaqiao University

Xiamen, China

whole rhythm, with the technique of "Yin, Ta, Guan, Dian, Zha" in the lubricating tune, based on skeleton of playing Pipa. "Finger, spectrum" of Nan Yin express and complete the great ensemble with the art of lubricating tune.

The difficulty is collecting Nan Yin, because traditional Nan Yin music resources are extraordinarily abundant but less old music were saved. There are too much tracks to collect, which includes thirteen sets of instrument music, two or three thousand vocal music, and forty-eight sets of "Finger set" which combined instrument music and vocal music. At the same time, a lot of accomplished artists died, with less old music remained. The remained paper usually passed in manuscript way and were hidden in various places in danger of damaged by worm and even vanish.

2) Build standard Nan Yin opern database with image encoding, image decoding, matching and identification: First Nan Yin opern digital system completes digital expressing and encoding special Nan Yin music learning mature digital opern technology[6]. Then it recognized opern with image recognition technique and transcribes opern into digitized code for long-term storage, transmission and inheritance. Finally it decodes digital Nan Yin music to play.

There are some improved image recognition method for low quality and high rate of error identification because of bad preservation[7]. When we build Nan Yin music database and input data in it, we need define data formats, type and connection, thinking about of storing format, sorting order and multiple retrieved possibilities[2].

3) Amendment and correction of opern with statistical models of Nan Yin opern: The remained paper usually passed in manuscript way with some phenomena of indistinct writing or wrong copying. While some Nan Yin music was passed by word of mouth which was incomplete even there was no opern. Besides there will be mistakes because of the long time. So before we store the digital collected Nan Yin opern or voice, we need to check for any errors, and if there are any, fix it.

Nan Yin digital system extracts features of Nan Yin opern, and digs the intrinsic cultural patterns, the rules, the structures, technical features and knowledge discovery. Check if a new opern is Nan Yin based on if it matching the structures and features of Nan Yin. And check if there are any errors based on if there are any outliers. Create statistical models of Nan Yin opern using the technologies of content-based audio features extracting [8], structure matching, association mining, structure mining, model mining, semantic mining. Then Fix the incomplete opern and correct errors in the opern using statistical models of Nan Yin opern.

\section{B. Nan Yin Audio Digital System}

1) Features, present situation and problems of Nan Yin audio: According to the survey, just about 20 musicians who can sing and play instrument over the age of 65 exist in the Quanzhou Nan Yin community[9]. Few sound records were remained and lack written records of Nan Yin theories and experience. Besides, different musicians in different schools show the different artistic styles on pronunciation, tonguing, lubricating tune, so standard of Nan Yin performance is multiple.

Traditional music resources of Nan Yin are plentiful. We need to find performance groups of Nan Yin and collect the voice of all the standard groups playing as complete as possible. Then organize and study the voice, concluding do 
some research, compare with the others, and correct errors, to ensure the comprehensive and accuracy before encode the voice.

2) Build standard Nan Yin opern database with voice encoding, voice decoding, matching and identification: The Nan Yin performance digital system completes digital expressing and encoding special Nan Yin music, learning mature digital audio technology[10]. Then it recognized audio with audio recognition technique and transcribes audio into digitized code[11] for long-term storage, transmission and inheritance. Finally it decodes digital Nan Yin music to play.

There are some difficult and special problems in digital encoding and decoding. Traditional Gongche notation have three components: voice coding, phalanx(the mark of chronaxy and playing Pipa). The marks of Gongche notation imply lubricating tune and skeleton, with which a player impromptu sing regularly, so one music is different from different musicians or at different times. Besides, because of the particularity Nan Yin's tune, structure, style, diapason are other than mainstream music so that the technology of Nan Yin feature extracting need to be improved based on Nan Yin's features[12]

3) Automatic assessment and exchanging between Nan Yin and mainstream music with similarity measurement: Nan Yin digital system extracts features of Nan Yin audio, and digs the intrinsic cultural patterns, the rules, the structures, technical features and knowledge discovery. Check if a new audio is Nan Yin based on if it matching the structures and features of Nan Yin. And check if there are any errors based on statistical models of voice.

It is hard to judge if it is good audio. We define the audio playing by the best musician as standard in the system, then build automatic assessment system by computing the similarity between new music and standard music with similarity measurement.

During promoting and inheriting, on one hand it need to highlight the historic feature and local feature, on the other hand communicate with mainstream music to promote development and innovation. We can achieve converting opern into audio and voice synthesis and complete the exchanging between Nan Yin and mainstream music through the technology of voice synthesis.

\section{Nan Yin Opern Auto-Play System}

1) Build opern-voice matching library with digital matching technology: The remained operns are incomplete and the remained audio-visual information is limited. There were many issues to be resolved, about playing opern or turning audio into audio automatically. Build opern-voice matching library by matching when the audio and the opern are all belong to a same music.

2) Automatic digital playing of Nan Yin opern with the technology of voice synthesis: After sort the corresponding voices in opern-voice matching library for opern, play it automatically with the technology of voice synthesis.

\section{Constructing the Map of Nan Yin Digital Cultural}

Knowledge and Spatial Semantic Model by Using

Ontology

Nan Yin contains rich expressing factors of cultural space. On the premise of keep its perfect classics respect its own artistic law and develop it reasonably. Based on ontology modeling, with the association analysis and similarity measure between Nan Yin and other classical dramas, it inherits traditional culture and enlarge Nan Yin's map of digital cultural knowledge.

Construct Nan Yin's spatial semantic model with opern by using ontology and self extend knowledge map of user models After extracting features and digging the intrinsic cultural patterns, the rules, the structures, technical features, it demonstrate the explicit and implicit spatial relationships among "Finger, spectrum, tune" types, bonds, musicians' position, music components, sequence, instrument rules and so on. The system expresses Gongche notation using ontology. And on this basis we dig the intrinsic cultural patterns, the rules, the structures, technical features and knowledge discovery with association mining, structure mining, model mining and semantic mining, then complete Nan Yin's map of digital cultural knowledge and spatial semantic model.

\section{CONCLUSION}

The System sort Nan Yin culture scientifically, fully and systematically and construct cultural knowledge models to store and manage it.

Nan Yin digital system play the model role of protecting and inheriting any special other music, languages, words and even minority cultures.

\section{ACKNOWLEDGEMENT}

In this paper, the research was supported by the Science and technology plan Foundation of Fujian Province of China(No.2013H1007), National Natural Science Foundation of China(Grant No.51305142), the Natural Science Foundation of Fujian Province of China(No.15141112) and the General Financial Grant from the China Postdoctoral Science Foundation(Grant No.2014M552429).

\section{REFERENCES}

[1] Junjie Yang. Discussion of audio digital protection and salvage of opera culture legacy[J].Home of dramas,2012,01:17-18+50

[2] Ni Zhong. The mode of original ecology music digital protection in the northweast of Hubei[J]. Shenzhou, 2013,02:198.

[3] Rui Niu. Compatibility and Development of Audio Digital Technology[J].Science and technology information,2010,16:645-646.

[4] Jingfang Yuan, Exploration and innovation of combination of ancient Chinese music culture and modern science and technology--concerning the significance and value of building "database of Chinese Guqin music culture"[J]. Central music university,2009,04:31-36.

[5] Feasibility of national science and technology program "key technologies and demonstration of music digital services" is proved[J]. Chinese science and technology information,2009,14:8

[6] Weilan Wang.The building library of Tangka image information resource

[7] JAIN AK,VAILAYA A.Shape-based retrieval:a case study withtrademark image databases. Pattern Recognition . 1998

[8] Wei Wang. Study on content-based audio retrieval feature extraction technology[D].Shanghai Jiao Tong University ,2008.

[9] Jinhua Chen,Zhibin Zhuang.Study on tourism development of nonmaterial cultural heritage based on RMP analysis--in the case of Quanzhou Nanyin[J].Leshan teachers college journal. 2007,05:42-44.

[10] Songdi Gao,Changle Zhou.Digital preservation and inheritance of Guqin cultural heritage.Mind and Calculations, 2010,04

[11] Ming Wang.Digital code of voice signals[J].Technological innovations and applications,2013,35:15-16.

[12] Bo Hui.Research and implement of speech recognition algorithm and feature extraction algorithm[D].Northwestern University,2008. 